\title{
Otolaryngologic Manifestations of Gastroesophageal Reflux
}

\author{
Michael Yim, $M D^{1}$ \\ Eric H. Chiou, $M D^{2,3}$ \\ Julina Ongkasuwan, MD FAAP FACS ${ }^{1,4, *}$
}

\author{
Address \\ *,1 Bobby R. Alford Department of Otolaryngology Head and Neck Surgery, Baylor \\ College of Medicine, Texas Children's Hospital, Houston, USA \\ Email: julinao@bcm.edu \\ ${ }^{2}$ Department of Pediatrics, Baylor College of Medicine, Houston, USA \\ ${ }^{3}$ Department of Pediatric Gastroenterology, Texas Children's Hospital, Houston, \\ USA \\ ${ }^{4}$ Division of Pediatric Otolaryngology, Texas Children's Hospital, Houston, USA
}

Published online: 9 July 2016

(C) Springer International Publishing AG 2016

This article is part of the Topical Collection on Otolaryngology

Keywords Gastroesophageal reflux disease $\cdot$ Pediatric otolaryngology $\cdot$ Extraesophageal $\cdot$ Laryngopharyngeal reflux

\section{Opinion statement}

Gastroesophageal reflux disease (GERD) is a common problem that has been linked to multiple extraesophageal manifestations relevant to the otolaryngologist. Finding evidence for a causal relationship between reflux and manifestations of otolaryngologic disease is often difficult, however, due in part to the non-specific nature of extraesophageal symptoms and the lack of pathognomonic endoscopic or laryngoscopic signs. This poses an even greater challenge in pediatrics given the inherent unreliability when obtaining subjectively reported data from younger aged children. Numerous studies have drawn a correlation between GERD and specific extraesophageal symptoms such as otitis media, sinusitis, chronic cough, and various laryngeal findings; however, determining a clear causative relationship has proven to be much more difficult. Further studies must be done to elucidate the true pathophysiologic mechanisms behind these disease processes. Diagnosis of GERD is challenging and is typically over-diagnosed among otolaryngologists based on laryngoscopic findings. This method has been proven to be both inconsistent and non-specific when evaluating for the presence of GERD. Impedance monitoring is supplanting $\mathrm{pH}$ probes as the new gold standard; the clinical utility of impedance testing among patients with extraesophageal symptoms remains unclear however and there are practical limitations in performing these studies in children. The accuracy of diagnostic tests (laryngoscopy, endoscopy, and $\mathrm{pH}$ - or $\mathrm{pH}$-impedance monitoring) for patients with suspected extraesophageal manifestations of gastroesophageal reflux disease remains suboptimal at this time. Management options for GERD include lifestyle changes, 
pharmacologic therapy, and surgical intervention. $\mathrm{H} 2$ blockers and proton pump inhibitors are effective treatment options in children but must be used judiciously given their potential side-effect profiles. Surgical options remain for those cases which are refractory to medical management. Multidisciplinary approaches and close collaboration between otolaryngologists and gastroenterologists is recommended to ensure application of best practice guidelines and for continued improvements in this area.

\section{Introduction}

Gastroesophageal reflux disease (GERD) is a common condition which has been studied extensively over the years with an abundance of published literature on the subject. Of particular interest to the otolaryngologist are the extraesophageal manifestations of GERD. Many signs and symptoms of upper aerodigestive disease such as hoarseness, laryngitis, rhinorrhea, sinusitis, globus sensation, recurrent croup, laryngomalacia, stridor, subglottic stenosis, otalgia, and dysphagia have all been previously associated with GERD in children [1]. Finding evidence for a causal relationship between reflux and manifestations of otolaryngologic disease is often difficult however, due in part to the non-specific nature of extraesophageal symptoms and the lack of pathognomonic endoscopic or laryngoscopic signs. Understanding the role of GERD in children necessitates a deeper understanding of the natural history and pathophysiology of the disease.

To start off, it is important to delineate physiologic reflux versus GERD and to review specific definitions for each. Physiologic reflux, or gastroesophageal reflux (GER), is the retrograde passage of gastric contents into the esophagus with or without regurgitation and vomiting. This is a normal process that occurs several times a day, usually postprandially of short duration,

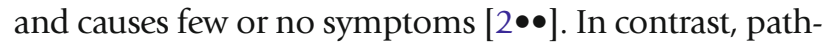
ologic reflux, or GERD, is defined as being present when there are troublesome symptoms and/or complications secondary to reflux of gastric contents. This definition was developed through a modified Delphi process in 2006 from a panel of worldwide experts who established the "Montreal definition and classification of GERD" [3], and a similar process occurred in 2009 specifically for pediatrics which ultimately resulted in the same definition [4]. Typical esophageal symptoms of reflux include heartburn, chest pain, and regurgitation. When referring to atypical GERD symptoms located outside of the esophagus, the phrase extraesophageal reflux is used, although other terms such as laryngopharyngeal reflux (LPR) and supraesophageal reflux (SER) have also been employed. The Montreal classification specifically emphasized that although an association between GERD and otolaryngologic manifestations exists, these extraesophageal syndromes are usually multifactorial and, furthermore, rarely occur in isolation without concurrent esophageal symptoms.

There are two main theories that have been proposed to explain the pathophysiology of extraesophageal symptoms of GERD, based on the action of direct microaspiration into the proximal airway versus vagally mediated reflexes (colloquially referred to as the "reflux" and "reflex" theories, respectively). The first mechanism involves gastric contents that directly stimulate the pharynx or larynx, with refluxate bypassing multiple anatomic boundaries which include the lower esophageal sphincter (LES), esophageal reflexes, the upper esophageal sphincter (UES), and ultimately the esophagoglottic closure reflex to subsequently cause tissue injury to acid sensitive mucosa [5]. The exact mechanism of refluxate bypassing this ensemble of protective mechanisms has remained controversial however, and there is a lot of ongoing research in this area. The second mechanism arises from the common embryonic origin between the esophagus and bronchial tree and subsequent shared neural pathway. Acidification of the distal esophagus may induce a vagally mediated arc which results in symptoms of chest pain and cough, possibly through reflexive bronchoconstriction. It is currently unknown if direct effect from gastric contents, vagally mediated reflexes, or a combination of factors contribute to development of extraesophageal symptoms [5].

\section{Clinical history}

Obtaining a clinical history for the purposes of diagnosing GERD in children presents a unique set of difficulties compared to adults. One caveat when assessing GERD in pediatrics is the inherent unreliability when obtaining subjectively reported data from younger aged children, and thus, the determination of whether a symptom is 
troublesome by self-report typically cannot be applied to children less than 8 years of age. Multiple studies have also shown that symptoms of GERD vary by age, with food refusal, regurgitation, and abdominal pain more severe in younger children while cough, epigastric pain, and vomiting are the predominant symptoms in older children [4]. It is also important to note that while GERD is frequently associated with a variety of aerodigestive symptoms, correlation does not necessarily equal causation, and as such making a diagnosis of GERD may not always be possible based on clinical symptoms alone. Given the above limitations, the main goal of obtaining a thorough history is to exclude other more concerning etiologies prior to pursuing further objective evaluation of GERD.

The differential for regurgitation, irritability, and cough is wide and includes common etiologies such as food allergies and colic. One important condition to keep in mind is eosinophilic esophagitis (EoE), which is a chronic inflammatory disorder of the esophagus characterized by dense eosinophilic infiltration and with symptoms nearly indistinguishable from GERD, especially in younger children. EoE is often times initially misdiagnosed as GERD and only appropriately identified on histopathology following failed reflux therapy [6]. The pathophysiology of EoE is poorly understood, but given its correlation with other IgE-driven atopic diseases such as allergic rhinitis, asthma, and atopic dermatitis, it is thought to arise from immune dysregulation. Patients with EoE have also been shown to have significant extraesophageal symptoms, particularly manifesting in the airway as cough, wheezing, asthma, rhinitis, sinusitis, nasal congestion, and even in one case as subglottic stenosis [7]. Recent work has shown that laryngeal eosinophils may be potentially utilized as a marker for chronic inflammation in patients with aerodigestive dysfunction, and there appears to be a correlation with the presence of EoE as well [8].

Another rare but often overlooked entity worth mentioning is the Sandifer syndrome. Initially described in the 1960s and named after neurologist Paul Sandifer, it involves spastic torticollis and dystonic upper body movements along with one or more of GERD, esophagitis, or a hiatal hernia. The pathophysiology is unclear; however, it is postulated that the abnormal posturing provides relief from the abdominal discomfort caused by acid reflux [9]. It is typically seen in infancy until early childhood with peak prevalence around 24 months of age, and appropriate management of GERD along with expected growth and development typically results in resolution of symptoms.

\section{Otolaryngologic manifestations}

It is important to understand the intricate relationships of the upper aerodigestive system and the pathophysiologic mechanisms that arise thereof in order to have a greater appreciation of the role GERD can play in various otolaryngologic disease processes.

\section{Otitis media}

Recent studies have implicated GERD as a possible contributing factor to the development of otitis media. Pepsin is a digestive protease and key deleterious component of gastric refluxate. The detection of pepsin and its precursor pepsinogen in airway specimens has been proposed as a biomarker for extraesophageal reflux [10]. One of the first studies to link the two disease processes discovered 1000 times higher concentrations of pepsin and pepsinogen in middle ear effusions compared to normal serum values, thus excluding simple plasma exudate as a possible source of this phenomena [11]. Expanding on this, when comparing pathologic samples from adenoid hypertrophy without middle ear disease versus adenoid hypertrophy with middle ear effusions, the latter group was found to have a significantly higher pepsinogen concentration collected from the adenoid tissue. Additionally, pepsinogen was detected in $84 \%$ of middle ear effusions from the same group [12]. These findings were further corroborated by another study that had similar results to the above, but additionally noted that pepsinogen mRNA was not detected in either group, indicating that the detected pepsinogen protein was not originally produced in the adenoid but originated from other sources. This same study also compared middle ear fluid from patients with otitis media with effusion versus patients undergoing cochlear implantation as a normal control and found higher concentrations of pepsin and pepsinogen in the diseased ears, again suggesting a correlation between GERD and otitis media [13]. Pepsin and pepsinogen are not without limitation however as there is a lack of normative values and assay standardization, and additional controlled studies are necessary to prove that reflux is solely responsible for the presence of these biomarkers outside of the GI tract. Given the above, further work needs to be done to demonstrate proof of cause and effect between extraesophageal reflux and middle ear inflammation. 


\section{Chronic rhinosinusitis}

Despite GERD and chronic rhinosinusitis (CRS) being common entities, they appear to occur in conjunction more often than simple coincidence, thus suggesting a potential link between the two disease processes [14]. Two potential pathophysiologic mechanisms have been described: direct effect from refluxate contacting the nasal cavity, and the presence of a nasal-esophagus reflex via the autonomic system [15]. The majority of published studies to date have not been able to definitely draw a causational relationship between the two despite a variety of different study formats, including usage of a multichannel $\mathrm{pH}$ probe, detection of pepsin and pepsinogen levels from nasal mucosa, PCR assay of $H$. pylori in nasal tissue, acid infusion testing, and even empiric use of proton pump inhibitors with symptom monitoring [16]. Anti-reflux medicine can be considered as an adjunctive treatment for patients with symptoms of both CRS and GERD; however, further studies need to be conducted in order to elucidate the true association between the two entities.

\section{Laryngeal manifestations}

Similar to the previous topics already discussed, evidence points to the likely connection between GERD and airway abnormalities. Proof of a cause and effect relationship, however, has yet to be fully elucidated [17]. A multitude of studies have described airway findings characteristic of GERD patients, including supraglottic and postglottic erythema and edema, laryngomalacia and even subglottic stenosis [18]. Laryngomalacia (LM) is the most common cause of stridor in infants, and it is often frequently linked with GERD. There are three types of LM: prolapse of the mucosa overlying the arytenoid cartilages, shortened aryepiglottic folds, and posterior displacement of the epiglottis [19]. It is important to understand the three different pathologies of LM as its relationship to GERD likely varies significantly with type. The causal relationship between the two is unclear, as some studies provide evidence that GERD is secondary to LM while others support the opposite viewpoint. GERD leading to LM is thought to be secondary to changes in airway resistance from supraglottic edema, whereas the latter perspective believes that LM leads to GERD by increased work of breathing altering the normal intrathoracic pressure gradient which is normally protective against reflux [19]. A systematic review of 27 studies provided some insight to the relationship as there is evidence for increased incidence of reflux in severe versus mild LM, improvement of LM-related symptoms with anti-reflux therapy, and histological evidence of reflux-related laryngeal inflammation in patients with LM [20]. It is important to note that particularly in the absence of established GERD however there is no evidence to support the routine use of reflux medications for LM.

GERD has also been previously implicated as a cofactor in the development of vocal nodules, and a recent systematic review found vocal fold edema and nodules to have the strongest correlation to GERD compared to other airway findings. The diagnosis of GERD was variable between studies and included esophageal biopsies, barium reflux on a barium swallow test, and $\mathrm{pH}$ probes. A moderated risk ratio analysis performed showed the percentage chance that a patient had GERD in the presence of specific endoscopic airway findings, with subglottic stenosis (65\%), supraglottic collapse (67\%), posterior glottis edema and erythema (70\%), and vocal fold edema and/or nodules ( $88 \%$ ) all found to have correlation [18]. With that said, the classic pathophysiology of vocal fold nodules describes it as bilateral thickening of the membranous folds arising from phonotraumatic voice use patterns [21], and as such the relationship between nodules and GERD is more likely an indirect rather than a direct one. One such etiology could be increased throat clearing and coughing secondary to reflux.

Chronic cough is a complex refractory condition attributable to a variety of pulmonary and extrapulmonary etiologies. One of the most common hypotheses for chronic cough is of course GERD. The pathophysiology has been proposed to be either vagal irritation through the esophagobronchial reflex pathway, direct aspiration of refluxate, or a combination of both. One study described the rate of cough increasing by $47 \%$ if immediately preceded by an episode of reflux as measured by $\mathrm{pH}$-impedance probe, which was a stronger response compared to cough increasing from antecedent phonation [22]. A retrospective review identifying common causes of chronic cough had GERD as one of the top three causes in addition to infection and airway hyper-reactivity [23]. In this particular study, however, the diagnosis of GERD was based on history and physical 
exam findings from laryngoscopy only; the limitations of which will be discussed further in the following section.

\section{Diagnostic testing}

\section{Laryngoscopy}

Given the inherent limitations of assessing reflux symptoms in infants based on subjective complaints, laryngeal examination is commonly utilized as the next step to assess for the presence of extraesophageal manifestations of GERD. A reflux finding score for infants (RFS-I) was developed from the adult reflux finding score (RFS) in order to have an objective measure of signs of extraesophageal reflux based on flexible or rigid laryngoscopy. This is a scoring system of eight different laryngeal findings ranging from excessive mucous to diffuse erythema and edema. Studies have shown this to have only fair to moderate interobserver reliability, however, and thus, given the poor reproducibility of results, usage of this measure is not recommended at this time in both adults and children [24, 25]. Overall, laryngeal findings on endoscopy are not specific and are subject to significant interrater variation of interpretation. This has likely contributed to the over-diagnosis of GERD among practicing otolaryngologists. One study investigating the prevalence of GERD through impedance probe testing in patients with a prior laryngoscopic diagnosis of LPR found a confirmed diagnosis of GERD in less than $40 \%$ of patients, illustrating the low specificity of laryngoscopy regarding extraesophageal reflux disease [26].

\section{Esophagoscopy}

Esophagoscopy and upper gastrointestinal endoscopy are additional methods of assessing structural changes secondary to GERD. When present, endoscopically visible breaks in the distal esophageal mucosa are a very reliable indicator of reflux esophagitis. Other findings such as mucosal erythema, pallor, and changes in vascular pattern are nonspecific and can sometimes be a

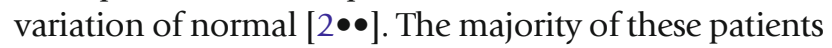
will not have endoscopic evidence of erosive esophagitis however, especially when taking empiric medical therapy for GERD. The overall yield of discovering findings such as esophagitis, Barrett esophagus, and other mucosal abnormalities in patients with suspected extraesophageal reflux is low and seems to add little to the overall diagnostic picture, and thus routine use of esophagoscopy and upper GI endoscopy for diagnosis of GERD as well as evaluation for extraesophageal symp-

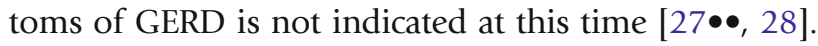
Endoscopy should be considered in those patients with concerning symptoms (hematemesis, unintentional weight loss, etc), patients at high risk for Barrett's esophagus, or in cases of failure to respond to appropriate medical therapy $[27 \bullet \bullet]$.

\section{PH Probes and impedance monitoring}

Of the various tests available for diagnosis of GERD, 24$\mathrm{h} \mathrm{pH}$ probe monitoring was for a long time considered the gold standard. Advances in instrumentation led to development of multichannel probes which allow for measurement of acid at multiple levels, with a drop of intraesophageal $\mathrm{pH}<4.0$ considered an acid reflux episode. The dual-probe $\mathrm{pH}$ monitor is the most wellknown example, with the proximal probe located either in the hypopharynx or upper esophagus. One limitation however is when the probe is located above the UES, they dry out and the measurements become unreliable. In order to overcome these issues the Restech $\mathrm{pH}$ measurement system was recently developed. The unique design allows consistent placement of the probe in the pharynx without drying of the catheter and this system has been proven to be superior compared to dual $\mathrm{pH}$ probe monitoring [29]. The Restech lacks a distal $\mathrm{pH}$ sensor however, and thus there are difficulties with correlating drops in oropharyngeal $\mathrm{pH}$ to reflux events. When comparing oropharyngeal $\mathrm{pH}$ monitoring to impedance monitoring, a high proportion of oropharyngeal events had no temporal correlation to actual reflux events, suggesting that oropharyngeal monitoring alone may overestimate the presence of extraesophageal reflux [30]. Unfortunately abnormal values from these methods do not necessarily correlate with severity of symptoms in infants, but normal monitoring in the setting of documented esophagitis can however suggest

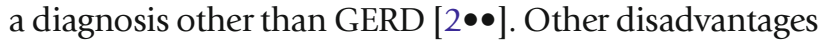
include the inability to detect non-acid reflux events and children pulling out the probe secondary to discomfort. As such this testing method has taken a backseat to the more recently developed combined multiple intraluminal impedance and $\mathrm{pH}$ monitoring system (MII). By measuring the changes in electrical impedance along the length of the esophageal catheter, it detects the passage of liquid, solid, gas, or mixed boluses. With this system, it is possible to measure non-acid reflux, and overall this has been shown to be superior to simple $\mathrm{pH}$ 
probe monitoring alone [31]. This analysis method has greater reproducibility but normal ranges for all ages have not yet been established and standardization is still in process [32•]. Given the lack of normative data, one of the main uses for MII testing in children is symptom correlation which does not rely on cutoff values for normal versus abnormal. Various methods have been attempted to quantify this relationship which includes the symptom index (SI), symptom sensitivity index (SSI), and symptom association probability (SAP) [31]. It is increasingly utilized in the setting of persistent reflux symptoms refractory to aggressive medical management; however, the clinical utility of impedance testing among patients with extraesophageal symptoms remains unclear, with evidence supporting a lack of association between the two [33-35].

\section{Esophageal manometry}

Esophageal manometry measures peristalsis and sphincter pressures and is useful to identify motor disorders of the esophagus that present very similar to GERD. It is neither sensitive nor specific for GERD, however, and

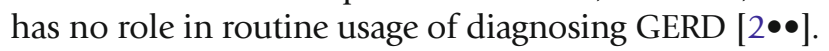
There are recent studies which suggest that abnormal esophagopharyngeal reflexes may be involved with extraesophageal reflux [36], but manometry should not be considered part of routine clinical evaluation at this time.

\section{Imaging studies}

Imaging studies such as barium contrast radiography, nuclear scintigraphy, and esophageal and gastric ultrasound do not have any routine role in diagnosing GERD in the pediatric population. An upper GI series is neither sensitive nor specific for GERD but can be used when the differential is broad and there is concern for an anatomic abnormality. Nuclear scintigraphy and ultrasound both have poor results when directly compared to $24-\mathrm{h}$ esophageal probe monitoring and are not indicated in children $[2 \bullet \bullet]$.

\section{Empiric medical therapy}

One last diagnostic method worth mentioning is empiric trial of proton pump inhibitors and observing for symptom improvement. While it is used in adults with moderate sensitivity and specificity, it has no role in young children and infants given the difficulty of assessing GERD based on symptom profile alone. Pharmacologic therapy in children should be strictly regulated to use in patients who have previously been diagnosed with GERD.

\section{Treatment}

Management options for GERD include lifestyle changes, pharmacologic therapy, and surgical intervention. For infants, there are a variety of strategies to decrease reflux including using hydrolyzed formula, smaller feedings, adding thickening agents, and positioning strategies such as placing the infant prone during postprandial awake periods. The effectiveness of each is variable, and positioning prone has challenges given its association with sudden infant death syndrome (SIDS). Thickening agents in particular have been shown to decrease overt regurgitation only without affecting the duration of acid $\mathrm{pH}$ in the esophagus [37]. There are no general lifestyle studies on children older than 1 year and current recommendations are simply extrapolations of adult strategies such as elevating the head of bed and sleeping in a lateral decubitus position. As of now, there is no evidence to support the routine elimination of any specific food for management of GERD in children $[2 \bullet \bullet]$.

Pharmacologic therapy is mainly comprised of histamine-2 (H2) receptor antagonists, proton pump inhibitors (PPI), and prokinetic agents. H2 blockers have been shown to be effective compared to placebo in large scale studies on adults [38], and there have been small case series in infants and children that have shown benefits in usage. Although there are no large randomized controlled trials specifically regarding the pediatric population, extrapolation of the data from large adult series as well as from the smaller case studies suggests that $\mathrm{H} 2$ blockers may be used in patients with GERD. Adverse effects from prolonged $\mathrm{H} 2$ blocker use can include rapid tachyphylaxis (reduction in response per dose) as well as irritability, headache, and head banging in some infants [39]. There is also an increased incidence of necrotizing enterocolitis with usage of $\mathrm{H} 2$ blockers in preterm infants [40].

Proton pump inhibitors are the mainstay in reflux therapy in both adults and children. Multiple large scale studies have validated the superiority of PPIs over H2 blockers, and the increased efficacy is due to their ability to inhibit meal-induced acid secretion [41]. Administration of long-term acid suppression is not currently recommended without a diagnosis given the potential side-effect profile. In addition, the largest multicenter RCT to date 
detected no difference between lansoprazole and placebo for symptoms attributed to GERD in infants aged 1-12 months, and no other placebocontrolled trial has demonstrated improvement in infants [42]. Given the above, there are currently no PPIs that have been approved for use in infants less than 1 year of age. There is limited data for PPI use in pediatrics, but at least in adults twice daily dosing of PPIs has been shown to be more effective than daily dosing in achieving clinical symptom response [43], although once daily dosing together with lifestyle modifications can sometimes lead to adequate acid suppression [44]. In addition, continuing lifestyle modifications for at least 6 months after the cessation of PPIs may be necessary to prevent early recurrence of symptoms [45]. Adverse effects from PPIs include headache, diarrhea, constipation, and nausea, and even increased incidence of acquired pneumonia and gastroenteritis has been reported [46]. Other side effects that have been reported in adults include changes in the gut microbiome; however, there are no studies that support this finding in children [47]. Increased risk of fracture has also been previously reported in adults on PPI; however, recent studies have not corroborated these findings and there is no clear mechanism as trials have shown no impact on calcium absorption nor have they been able to find any association with increased risk of osteoporosis [48]. Some studies have reported the association of gastric acid suppression with increased risk of community acquired and nosocomial pneumonia; however, the magnitude of effect is small and a few studies were poorly designed with significant confounders, bringing into question the accuracy of the results [49•].

Prokinetics such as cisapride, metoclopramide, domperidone, bethanechol, erythromycin, or baclofen do not have sufficient evidence to justify the use in children for GERD. A systemic review showed that although these agents may potentially be a viable treatment option, current literature available is inadequate to recommend their use in extraesophageal reflux [50]. In addition, multiple studies have shown the potential adverse effect profile (prolonged QT interval, extrapyramidal symptoms, seizures, etc) to be greater than the potential benefits for these medicines, thus leading to an unfavorable risk-benefit ratio. Other agents such as buffering agents, alginate, and sucralfate are useful for intermittent heartburn symptoms but the chronic use of these medicines is not currently advised $[2 \bullet \bullet]$.

\section{Surgery}

For patients with chronic relapsing GERD refractory to medical management, referral to a pediatric gastroenterologist is warranted for escalation of therapy and consideration for possible surgical intervention. Studies have shown improvement of extraesophageal manifestations of GERD following laparoscopic fundoplication [51-53], justifying the utilization of this intervention in patients with severe or refractory disease, although the percentage of patients who respond to surgery is less than those with classical GERD [54]. Heartburn with and without regurgitation and an esophageal $\mathrm{pH}<$ 4 for more than $12 \%$ of a 24 -h period are both significant predictors of extraesophageal symptom improvement following fundoplication [55].

\section{Conclusion}

GERD is a common problem that has been linked to multiple extraesophageal manifestations relevant to the otolaryngologist. It is important to be cognizant of the complex relationship of the upper aerodigestive system and the role that reflux may play in various otolaryngologic disease processes. The accuracy of diagnostic tests (laryngoscopy, endoscopy, and $\mathrm{pH}$ or $\mathrm{pH}$-impedance monitoring) for patients with suspected extraesophageal manifestations of gastroesophageal reflux disease remains suboptimal, however, and further studies need to be done to determine the best approach to identifying patients in whom 
treatment of reflux results in improvement of otolaryngologic complaints. Multidisciplinary approaches and close collaboration between otolaryngologists and gastroenterologists are recommended to ensure application of best practice guidelines and for continued improvements in this area.

\section{Compliance with Ethical Standards}

\section{Conflict of Interest}

Michael Yim, Eric H. Chiou, and Julina Ongkasuwan declare that they have no conflict of interest.

Human and Animal Rights and Informed Consent

This article does not contain any studies with human or animal subjects performed by any of the authors.

\section{References and Recommended Reading}

Papers of particular interest, published recently, have been highlighted as:

- Of importance

$\bullet \quad$ Of major importance

1. Gilger MA. Pediatric otolaryngologic manifestations of gastroesophageal reflux disease. Curr Gastroenterol Rep. 2003;5(3):247-52.

2.• Vandenplas Y, Rudolph CD, Di Lorenzo C, et al. Pediatric gastroesophageal reflux clinical practice guidelines: joint recommendations of the North American Society for Pediatric Gastroenterology, Hepatology, and Nutrition (NASPGHAN) and the European Society for Pediatric Gastroenterology, Hepatology, and $\mathrm{Nu}$ trition (ESPGHAN). J Pediatr Gastroenterol Nutr. 2009;49(4):498-547. doi:10.1097/MPG. 0b013e3181b7f563.

Most comprehensive and up-to-date resource for pediatric GERD practice guidelines.

3. Vakil N, van Zanten SV, Kahrilas P, Dent J, Jones R, Global Consensus Group. The montreal definition and classification of gastroesophageal reflux disease: a global evidence-based consensus. Am J Gastroenterol. 2006;101(8):1900-20.

4. Sherman PM, Hassall E, Fagundes-Neto U, et al. A global, evidence-based consensus on the definition of gastroesophageal reflux disease in the pediatric population. Am J Gastroenterol. 2009;104(5):1278-95. doi:10. 1038/ajg.2009.129.

5. Hom C, Vaezi MF. Extraesophageal manifestations of gastroesophageal reflux disease. Gastroenterol Clin North Am. 2013;42(1):71-91. doi:10.1016/j.gtc.2012. 11.004 .

6. Dauer EH, Ponikau JU, Smyrk TC, Murray JA, Thompson DM. Airway manifestations of pediatric eosinophilic esophagitis: a clinical and histopathologic report of an emerging association. Ann Otol Rhinol Laryngol. 2006;115(7):507-17.

7. Hill CA, Ramakrishna J, Fracchia MS, et al. Prevalence of eosinophilic esophagitis in children with refractory aerodigestive symptoms. JAMA Otolaryngol Head Neck Surg. 2013;139(9):903-6. doi:10.1001/jamaoto. 2013.4171.

8. Yawn RJ, Acra S, Goudy SL, Flores R, Wootten CT. Eosinophilic laryngitis in children with aerodigestive dysfunction. Otolaryngol Head Neck Surg. 2015;153(1):124-9. doi:10.1177/ 0194599815577568.

9. Lehwald N, Krausch M, Franke C, Assmann B, Adam R, Knoefel WT. Sandifer syndrome-a multidisciplinary diagnostic and therapeutic challenge. Eur J Pediatr Surg. 2007;17(3):203-6. doi:10.1055/s-2007-965145.

10. Samuels TL, Johnston N. Pepsin as a marker of extraesophageal reflux. Ann Otol Rhinol Laryngol. 2010;119(3):203-8.

11. Tasker A, Dettmar PW, Panetti M, Koufman JA, Birchall JP, Pearson JP. Reflux of gastric juice and glue ear in children. Lancet. 2002;359(9305):493.

12. Al-Saab F, Manoukian JJ, Al-Sabah B, et al. Linking laryngopharyngeal reflux to otitis media with effusion: pepsinogen study of adenoid tissue and middle ear fluid. J Otolaryngol Head Neck Surg. 2008;37(4):565-71. 
13. Luo HN, Yang QM, Sheng Y, et al. Role of pepsin and pepsinogen: linking laryngopharyngeal reflux with otitis media with effusion in children. Laryngoscope. 2014;124(7):E294-300. doi:10.1002/lary.24538.

14. Phipps CD, Wood WE, Gibson WS, Cochran WJ. Gastroesophageal reflux contributing to chronic sinus disease in children: a prospective analysis. Arch Otolaryngol Head Neck Surg. 2000;126(7):831-6.

15. Katle EJ, Hatlebakk JG, Steinsvag S. Gastroesophageal reflux and rhinosinusitis. Curr Allergy Asthma Rep. 2013;13(2):218-23. doi:10.1007/s11882-013-0340-5.

16. Hanna BC, Wormald PJ. Gastroesophageal reflux and chronic rhinosinusitis. Curr Opin Otolaryngol Head Neck Surg. 2012;20(1):15-8. doi:10.1097/MOO. 0b013e32834e8f11.

17. Mitzner R, Brodsky L. Multilevel esophageal biopsy in children with airway manifestations of extraesophageal reflux disease. Ann Otol Rhinol Laryngol. 2007;116(8):571-5.

18. May JG, Shah P, Lemonnier L, Bhatti G, Koscica J, Coticchia JM. Systematic review of endoscopic airway findings in children with gastroesophageal reflux disease. Ann Otol Rhinol Laryngol. 2011;120(2):116-22.

19. Olney DR, Greinwald Jr JH, Smith RJ, Bauman NM. Laryngomalacia and its treatment. Laryngoscope. 1999;109(11):1770-5. doi:10.1097/00005537199911000-00009.

20. Hartl TT, Chadha NK. A systematic review of laryngomalacia and acid reflux. Otolaryngol Head Neck Surg. 2012;147(4):619-26.

21. Tezcaner CZ, Karatayli Ozgursoy S, Sati I, Dursun G. Changes after voice therapy in objective and subjective voice measurements of pediatric patients with vocal nodules. Eur Arch Otorhinolaryngol.

2009;266(12):1923-7. doi:10.1007/s00405-0091008-6.

22. Francis DO, Slaughter JC, Ates F, et al. Airway hypersensitivity, reflux, and phonation contribute to chronic cough. Clin Gastroenterol Hepatol. 2015.

23. Cash H, Trosman S, Abelson T, Yellon R, Anne S. Chronic cough in children. JAMA Otolaryngol Head Neck Surg. 2015;141(5):417-23. doi:10.1001/ jamaoto.2015.0257.

24. Singendonk M, Pullens B, Van Heteren J, et al. Op-5 interobserver validity of the reflux finding score for infants (rfs-I) in flexible versus rigid laryngoscopy. J Pediatr Gastroenterol Nutr. 2015;61(4):510-1. doi:10. 1097/01.mpg.0000472209.38894.78.

25. Chang BA, MacNeil SD, Morrison MD, Lee PK. The reliability of the reflux finding score among general otolaryngologists. J Voice. 2015;29(5):572-7. doi:10. 1016/j.jvoice.2014.10.009.

26. De Bortoli N, Nacci A, Savarino E, et al. How many cases of laryngopharyngeal reflux suspected by laryngoscopy are gastroesophageal reflux disease-related? World J Gastroenterol. 2012;18(32):4363-70. doi:10. 3748/wjg.v18.i32.4363.

27.• ASGE Standards of Practice Committee, Muthusamy VR, Lightdale JR, et al. The role of endoscopy in the management of GERD. Gastrointest Endosc. 2015;81(6):1305-10. doi:10.1016/j.gie.2015.02.021. Solid overview and general practice guideline for the use of endoscopy in GERD.

28. Madanick RD. Extraesophageal presentations of GERD: where is the science? Gastroenterol Clin North Am. 2014;43(1):105-20. doi:10.1016/j.gtc.2013.11.007.

29. Worrell SG, DeMeester SR, Greene CL, Oh DS, Hagen JA. Pharyngeal $\mathrm{pH}$ monitoring better predicts a successful outcome for extraesophageal reflux symptoms after antireflux surgery. Surg Endosc. 2013;27(11):4113-8. doi:10.1007/s00464-0133076-3.

30. Chiou E, Rosen R, Jiang H, Nurko S. Diagnosis of supra-esophageal gastric reflux: correlation of oropharyngeal $\mathrm{pH}$ with esophageal impedance monitoring for gastro-esophageal reflux. Neurogastroenterol Motil. 2011;23(8):717-e326. doi:10.1111/j.1365-2982. 2011.01726.x.

31. Mousa HM, Rosen R, Woodley FW, et al. Esophageal impedance monitoring for gastroesophageal reflux. J Pediatr Gastroenterol Nutr. 2011;52(2):129-39. doi:10.1097/MPG.0b013e3181ffde67.

32. Yellon RF, Goyal A. What is the best test for pediatric gastroesophageal reflux disease? Laryngoscope. 2013;123(12):2925-7. doi:10.1002/lary.23656.

Provides a succinct summary of a difficult topic and compares the various modalities.

33. Roberts JR, Aravapalli A, Pohl D, Freeman J, Castell DO. Extraesophageal gastroesophageal reflux disease (GERD) symptoms are not more frequently associated with proximal esophageal reflux than typical GERD symptoms. Dis Esophagus. 2012;25(8):678-81. doi:10.1111/j.1442-2050.2011.01305.x.

34. Greifer $\mathrm{M}, \mathrm{Ng} \mathrm{K}$, Levine J. Impedance and extraesophageal manifestations of reflux in pediatrics. Laryngoscope. 2012;122(6):1397-400. doi:10.1002/ lary. 23250.

35. Kavitt RT, Yuksel ES, Slaughter JC, et al. The role of impedance monitoring in patients with extraesophageal symptoms. Laryngoscope. 2013;123(10):2463-8. doi:10.1002/lary.23734.

36. Babaei A, Venu M, Naini SR, et al. Impaired upper esophageal sphincter reflexes in patients with supraesophageal reflux disease. Gastroenterology. 2015;149(6):1381-91. doi:10.1053/j.gastro.2015. 07.007.

37. Khoshoo V, Ross G, Brown S, Edell D. Smaller volume, thickened formulas in the management of gastroesophageal reflux in thriving infants. J Pediatr Gastroenterol Nutr. 2000;31(5):554-6.

38. Khan M, Santana J, Donnellan C, Preston C, Moayyedi P. Medical treatments in the short term management of reflux oesophagitis. Cochrane Database Syst Rev. 2007;2:CD003244. doi:10.1002/14651858. CD003244.

39. Orenstein SR, Shalaby TM, Devandry SN, et al. Famotidine for infant gastro-oesophageal reflux: a multi-centre, randomized, placebo-controlled, 
withdrawal trial. Aliment Pharmacol Ther. 2003;17(9):1097-107.

40. Guillet R, Stoll BJ, Cotten CM, et al. Association of H2blocker therapy and higher incidence of necrotizing enterocolitis in very low birth weight infants. Pediatrics. 2006;117(2):e137-42.

41. Kahrilas PJ, Shaheen NJ, Vaezi MF, et al. American gastroenterological association medical position statement on the management of gastroesophageal reflux disease. Gastroenterology. 2008;135(4):1383-91. doi:10.1053/j.gastro.2008.08.045.

42. Orenstein SR, Hassall E, Furmaga-Jablonska W, Atkinson S, Raanan M. Multicenter, double-blind, randomized, placebo-controlled trial assessing the efficacy and safety of proton pump inhibitor lansoprazole in infants with symptoms of gastroesophageal reflux disease. J Pediatr. 2009;154(4):514520.e4. doi:10.1016/j.jpeds.2008.09.054.

43. Park W, Hicks DM, Khandwala F, et al. Laryngopharyngeal reflux: prospective cohort study evaluating optimal dose of proton-pump inhibitor therapy and pretherapy predictors of response. Laryngoscope. 2005;115(7):1230-8.

44. Reichel O, Keller J, Rasp G, Hagedorn H, Berghaus A. Efficacy of once-daily esomeprazole treatment in patients with laryngopharyngeal reflux evaluated by 24 hour $\mathrm{pH}$ monitoring. Otolaryngol Head Neck Surg. 2007;136(2):205-10.

45. Chappity P, Kumar R, Deka RC, Chokkalingam V, Saraya A, Sikka K. Proton pump inhibitors versus solitary lifestyle modification in management of laryngopharyngeal reflux and evaluating who is at risk: Scenario in a developing country. Clin Med Insights Ear Nose Throat. 2014;7:1-5. doi:10.4137/CMENT. S13799.

46. Canani RB, Cirillo P, Roggero P, et al. Therapy with gastric acidity inhibitors increases the risk of acute gastroenteritis and community-acquired pneumonia in children. Pediatrics. 2006;117(5):e817-20.

47. Freedberg DE, Lebwohl B, Abrams JA. The impact of proton pump inhibitors on the human gastrointestinal microbiome. Clin Lab Med. 2014;34(4):771-85. doi:10.1016/j.cll.2014.08.008.
48. Leontiadis GI, Moayyedi P. Proton pump inhibitors and risk of bone fractures. Curr Treat Options Gastroenterol. 2014;12(4):414-23. doi:10.1007/ s11938-014-0030-y.

49. Parikh N, Howden CW. The safety of drugs used in acid-related disorders and functional gastrointestinal disorders. Gastroenterol Clin North Am. 2010;39(3):529-42. doi:10.1016/j.gtc.2010.08.009.

Good overview of the potential side effects of $\mathrm{H} 2$ blockers and PPIs.

50. Glicksman JT, Mick PT, Fung K, Carroll TL. Prokinetic agents and laryngopharyngeal reflux disease: prokinetic agents and laryngopharyngeal reflux disease: a systematic review. Laryngoscope. 2014;124(10):2375-9. doi:10.1002/lary.24738.

51. Zhang C, Wang ZG, Wu JM, et al. A preliminary investigation of laparoscopic fundoplication treatment on gastroesophageal reflux disease-related respiratory symptoms. Surg Laparosc Endosc Percutan Tech. 2012;22(5):406-9. doi:10.1097/SLE. ob013e3182628913.

52. Rantanen T, Kiljander T, Salminen P, Ranta A, Oksala N, Kellokumpu I. Reflux symptoms and side effects among patients with gastroesophageal reflux disease at baseline, during treatment with PPIs, and after nissen fundoplication. World J Surg. 2013;37(6):1291-6. doi:10.1007/s00268-013-1979-8.

53. Koch OO, Antoniou SA, Kaindlstorfer A, Asche KU, Granderath FA, Pointner R. Effectiveness of laparoscopic total and partial fundoplication on extraesophageal manifestations of gastroesophageal reflux disease: a randomized study. Surg Laparosc Endosc Percutan Tech. 2012;22(5):387-91. doi:10. 1097/SLE.0b013e31825efb5b.

54. Iqbal M, Batch AJ, Spychal RT, Cooper BT. Outcome of surgical fundoplication for extraesophageal (atypical) manifestations of gastroesophageal reflux disease in adults: a systematic review. J Laparoendosc Adv Surg Tech A. 2008;18(6):789-96. doi:10.1089/lap.2007.0165.

55. Francis DO, Goutte M, Slaughter JC, et al. Traditional reflux parameters and not impedance monitoring predict outcome after fundoplication in extraesophageal reflux. Laryngoscope. 2011;121(9):1902-9. doi:10. 1002/lary.21897. 